\section{heinz hossdorf}

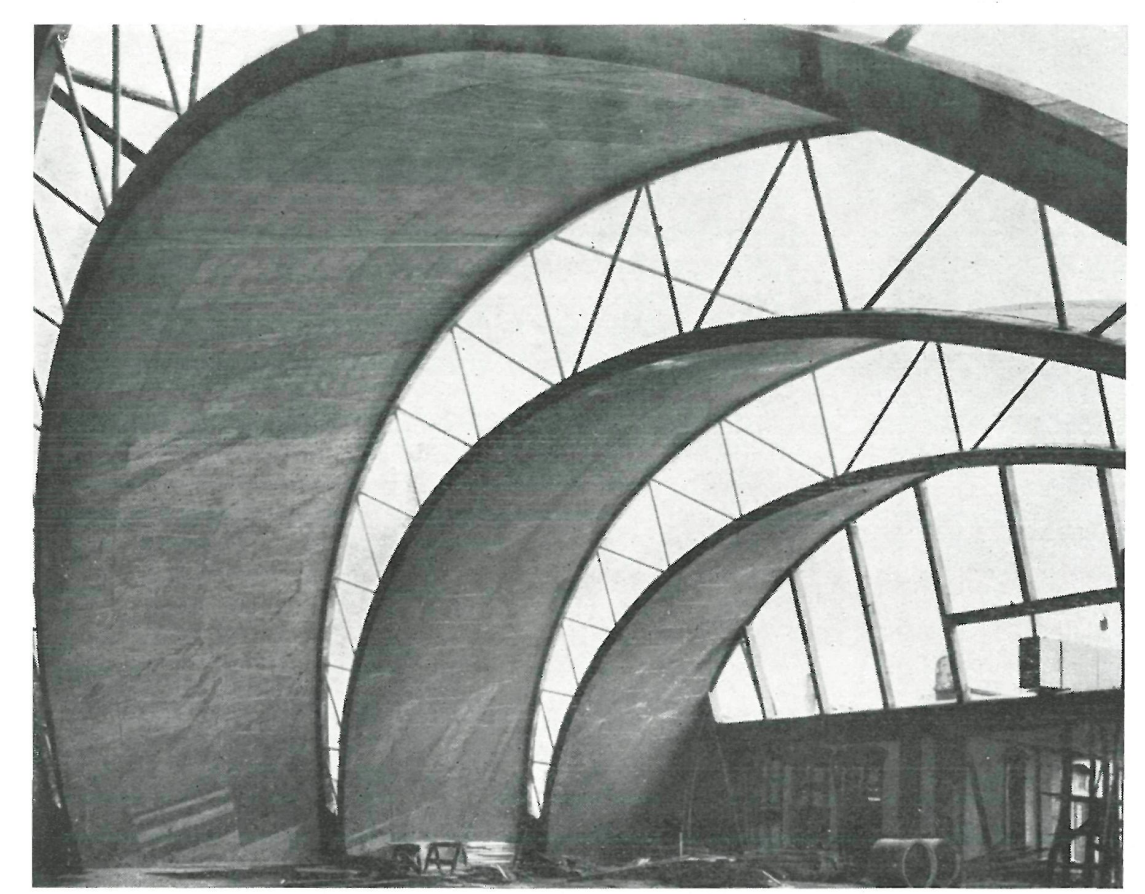

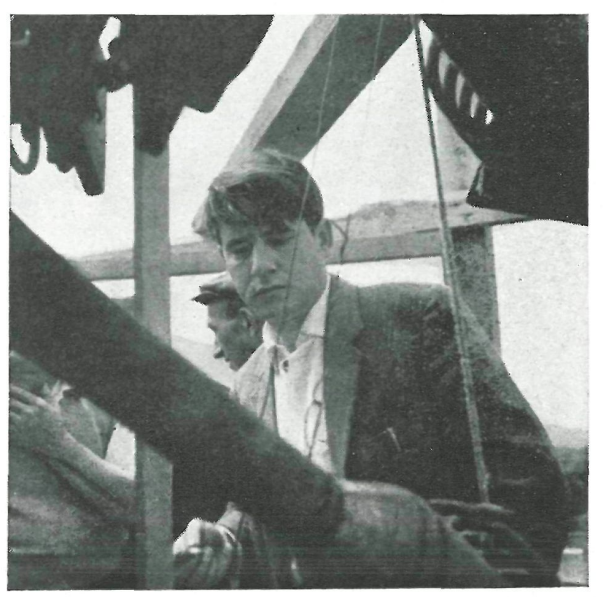

$103-1$

El ingeniero suizo Heinz Hossdorf, es ya familiar en las páginas de INFORMES. Hemos publicado varias de sus obras, en las que se acusa de forma destacada su personalidad. Sus cálculos, sus estructuras, sus ensayos sobre modelo reducido..., le han llevado a realizar frecuentes incursiones en el campo arquitectónico, obligándole al trato continuo y directo con un sinnúmero de arquitectos.

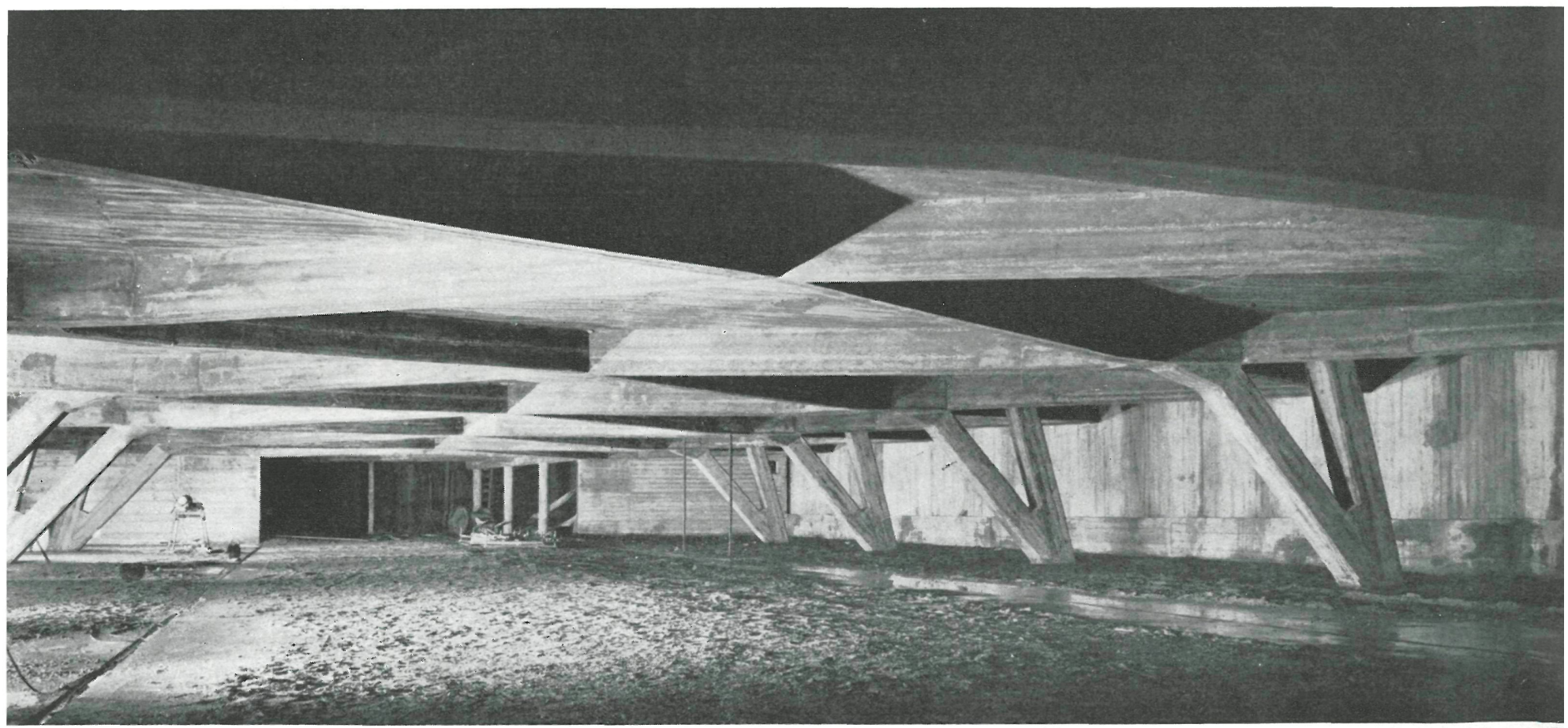




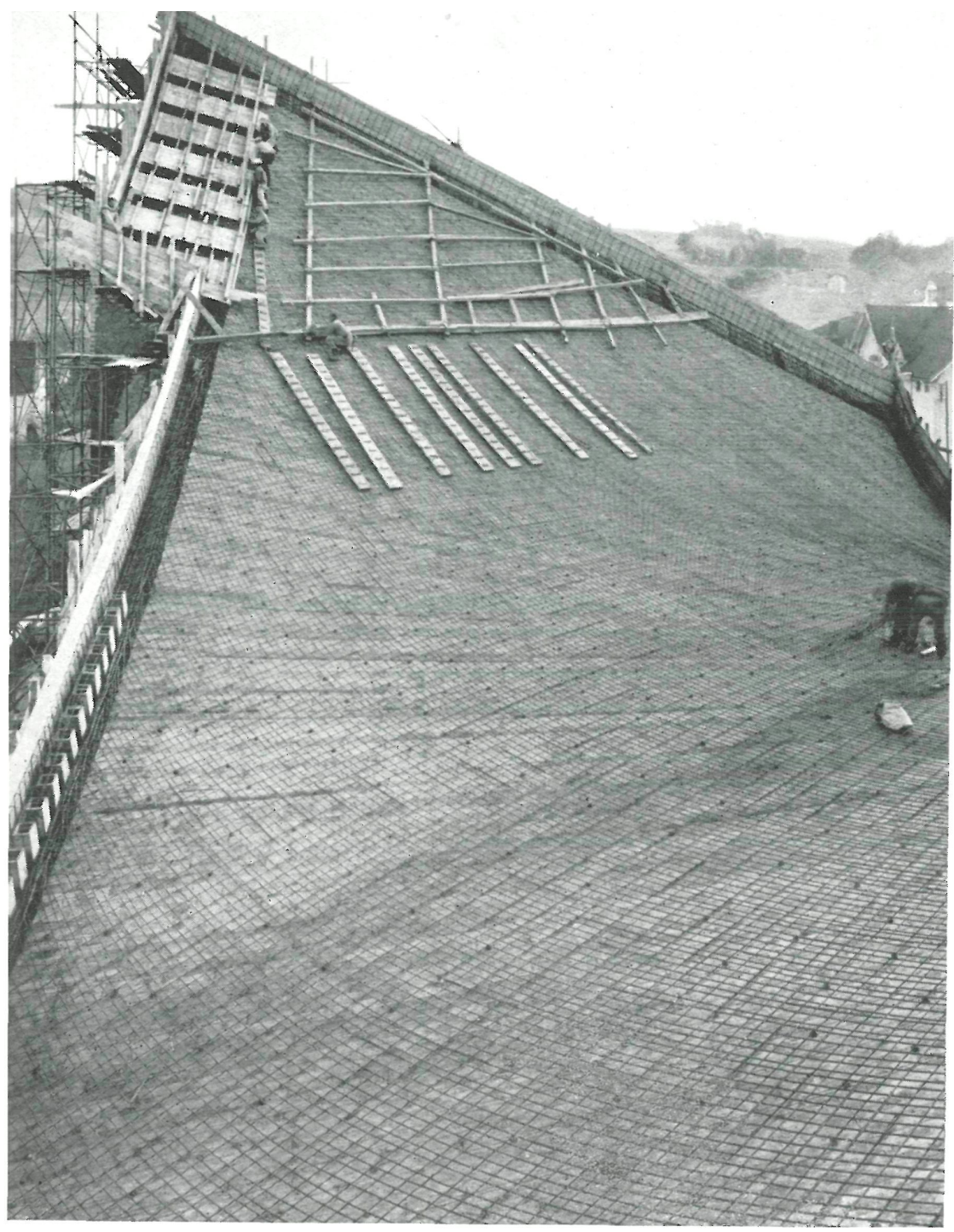

De este trato y de esta eficaz colaboración ha nacido una experiencia aleccionadora que le permite poder fijar claramente el papel de arquitecto e ingeniero en este trabajo en equipo, cada vez más necesario y frecuente, y que obliga a un mayor tecnicismo arquitectónico y a una menor rigurosidad matemática ingenieril. Ni falsas arquitecturas que ocultan y desprecian a algo tan fundamental como es su propia estructura, ni arquitecturas sólo expresión formal de ecuaciones diferenciales fácilmente integrables. El arquitecto debe ser cada vez más ingeniero y éste cada vez más arquitecto para que el lenguaje y la meta de su trabajo conjunto sean únicos.

Pese a ser sobradamente conocido, sirvan de presentación de Hossdorf algunas de sus obras:

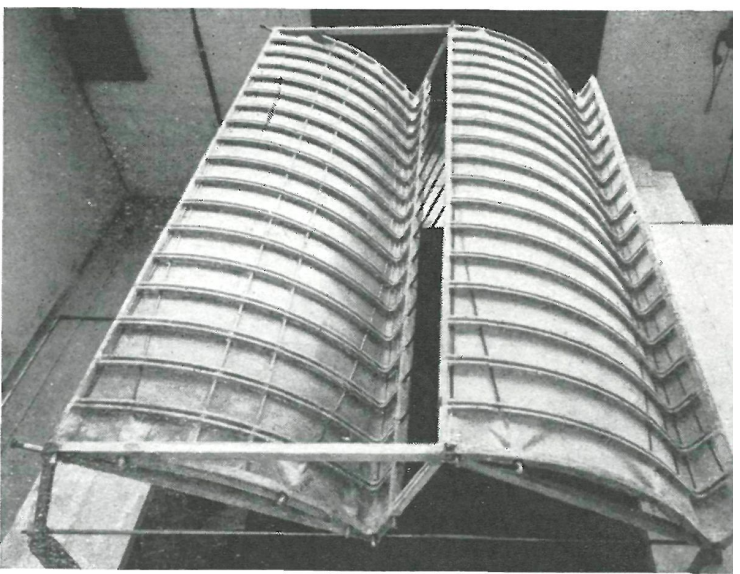

1. Estructura laminar de un edificio industrial en St. Gallen -Gummibandmeberei af Gossau-. Arquitectos: Danzeisen y Voser.

2. Aparcamiento subterráneo en Basilea-Zolli Garage-. Arquitecto: O. U. V. Sens.

3. Iglesia - Bruderklausenkirche en Winkeln, St. Gallen. Arquitecto: Ernest Brautschen. 
4. Iglesia en Vicques, Berner Jura. Arquitecto: Pierre Dumas.

5. Naves de estructura laminar con elementos prefabricados y pretensados para una cooperativa suiza.

Y una vez presentado, dejémosle hablar sobre un tema sugestivo:

\section{arquitectura e ingeniería, arte y técnica}

De forma premiosa, pero con rit-

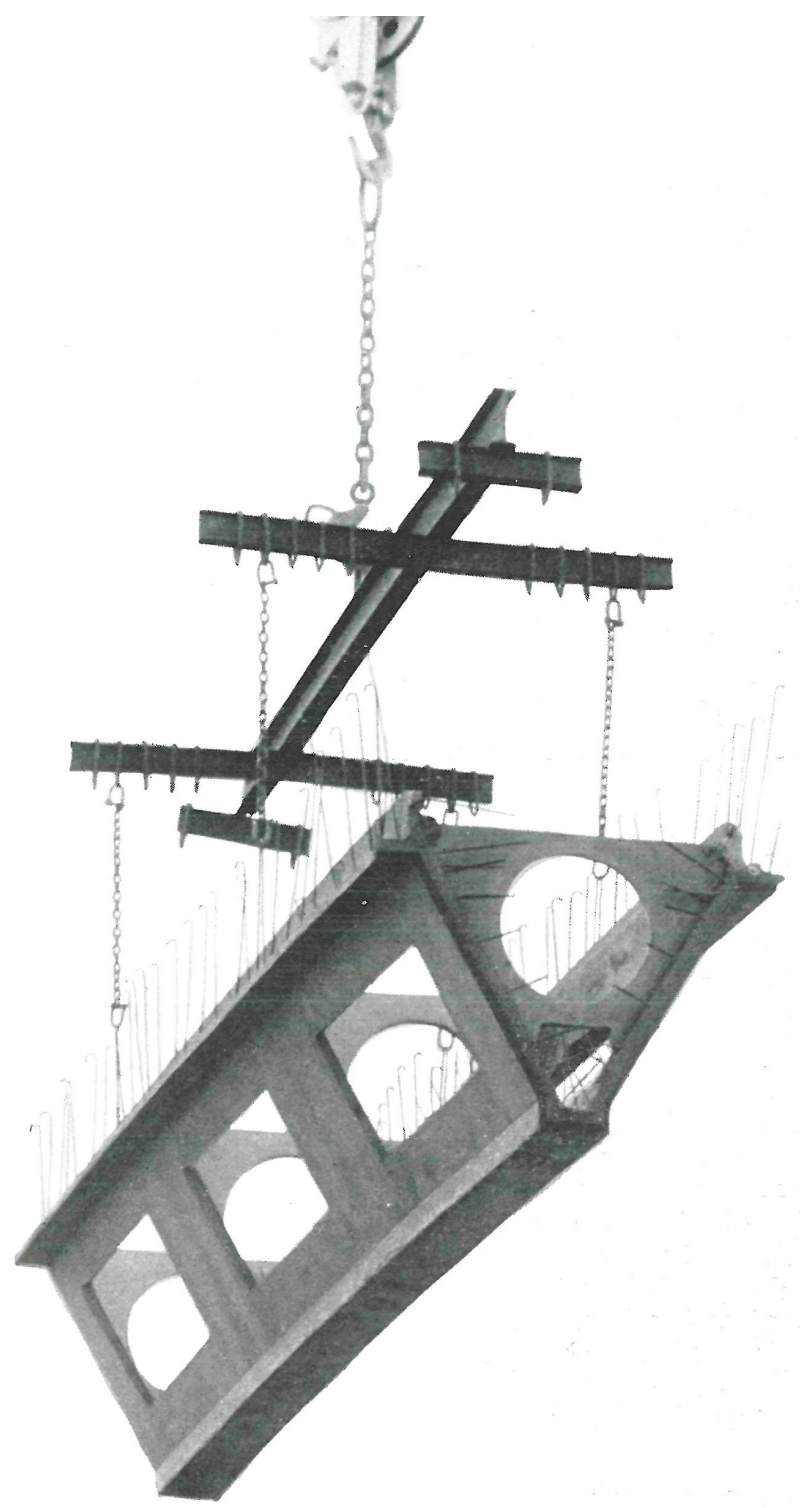
mo cada vez más acelerado, la técnica constructiva intenta seguir eI progreso general de las ciencias aplicadas. Demasiados intereses materiales e ideas tradicionales existen en el modo de hacer usual, muy difíciles de vencer para conseguir, como ocurre en otros campos de la técnica, que la técnica constructiva pueda experimentar un desarrollo explosivo.

Esta evolución continua de la técnica está estrechamente relacionada, y de forma automática, con un cambio continuo de la expresión arquitectónica condicionada a las limitaciones del material. Cada uno de los materiales y procedimientos que están en desarrollo poseen propiedades específicas, cuyo conocimiento exacto capacita al hombre para crear formas de expresión duradera que perduran sobre las variaciones de la moda. El concepto de lo moderno no puede limitarse a la valoración formal, como suele hacerse corrientemente por razones muy comprensibles; al contrario, este formalismo está frenando la investigación a lo verdaderamente moderno. La evolución del material, hormigón armado y pretensado, por ejemplo, se produce con tanta velocidad que no queda tiempo para la formación de un estilo en el sentido clásico. También los elementos constructivos que están al alcance del arquitecto están sometidos a este mismo proceso.

En la construcción actual, podemos decir que no hay "estilo" moderno. Lo característico en la expresión formal de nuestro tiempo no es el "estilo", sino más bien la foto instantánea del cambio continuo de posibilidades. En la expresión de este dinamismo se esconde posiblemente la única constante de nuestra arquitectura, y en ella habría que buscar el factor común de todas las construcciones de nuestro tiempo.

Como confirmación de esta teoría vamos a considerar sólo, a modo de ejemplo, la influencia de la evolución técnica de la construcción en hormigón armado sobre la expresión arquitectónica.

Evidentemente, la arquitectura no consiste sólo en "forma" y "técnica". No, la primera meta de un buen arquitecto creador es la de crear un espacio necesario al hombre para desarrollar una función; un espacio que en primer lugar no es más que una visión abstracta. 


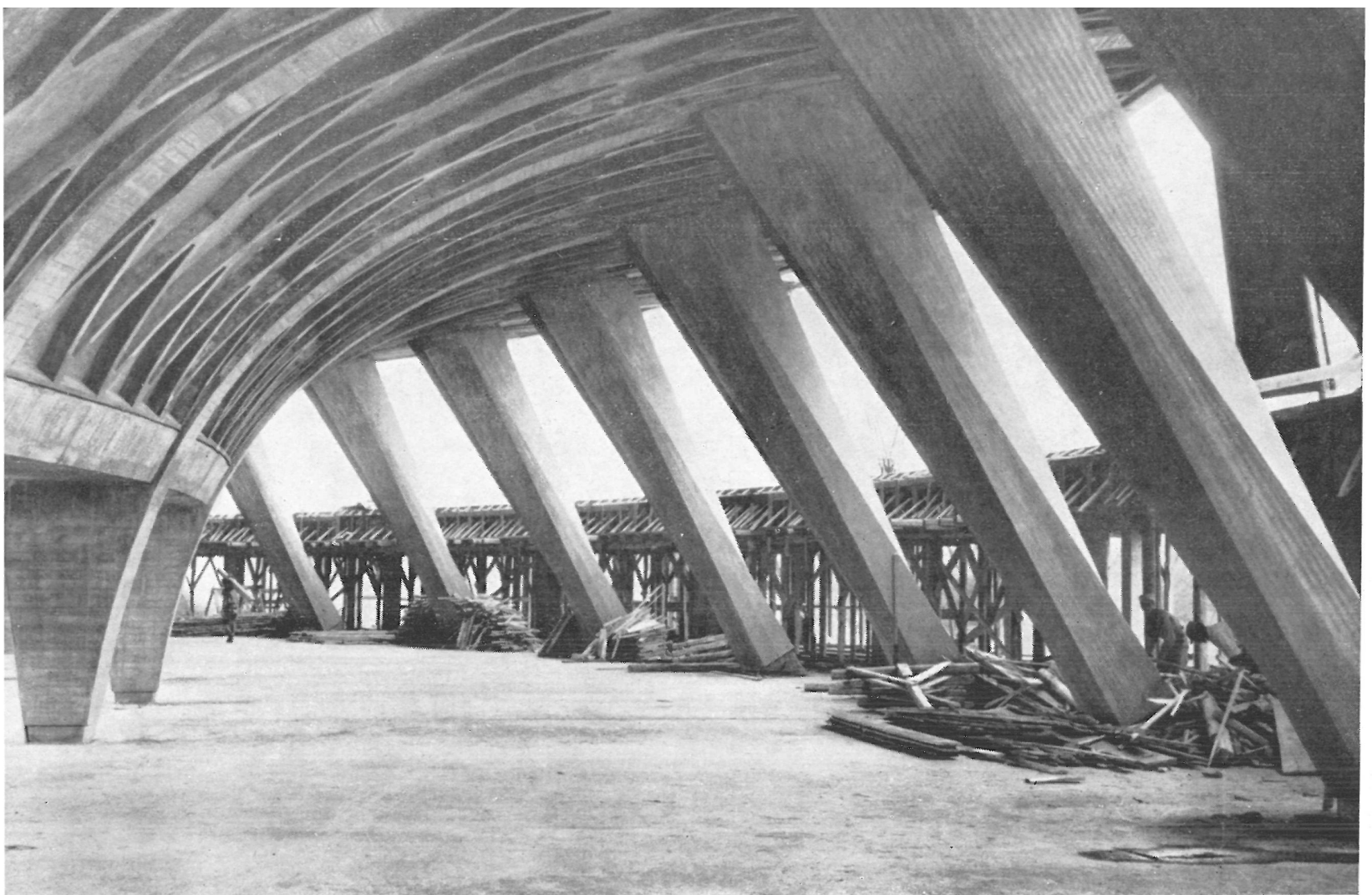

Pier Luigi Nervi: Sucesivas fases de construcción del Palacio de los De. portes de Roma

Después esta visión geométrica abstracta se materializa, es decir, damos al espacio una envoltura que cumple unas determinadas exigencias estéticas y técnicas. Sólo de esta segunda fase es de la que voy a tratar.

Parece completamente lógico y natural que el ingeniero debe ejercer notable influencia en la concepción de esta envoltura, ya que él es el técnico especializado que posee conocimientos suficientemente profundos sobre la naturaleza de los materiales de construcción, de sus formas idóneas en relación con la escala, de los métodos constructivos, dimensionamiento, economía..., que permiten al arquitecto crear las formas constructivas que agoten razonablemente las posibilidades de cada momento. Por eso, el aspecto formal de un edificio está influenciado de forma tajante por la técnica. De lo expuesto anteriormente se desprende que el ingeniero de hoy está obligado a superarse a sí mismo como hombre en este nuevo tema lleno de responsabilidades.

El ingeniero se debe preocupar de las consecuencias formales de sus conocimientos científicos. Tiene que darse perfecta cuenta que no tiene que realizar las ideas formales del arquitecto forzando las posibilidades naturales, sino desarrollar activamente proposiciones e ideas nacidas del conocimiento de las posibilidades técnicas. El arquitecto debe corregir su concepción visionaria en estrecha colaboración con el ingeniero, porque las posibilidades desbordadas que se han producido como consecuencia del rápido desarrollo de la técnica de la construcción, ocurrido en estas tres últimas décadas, no están para gastarlas sin fundamento. En la construcción, el ingeniero tiene el papel de encontrar y guardar la escala.

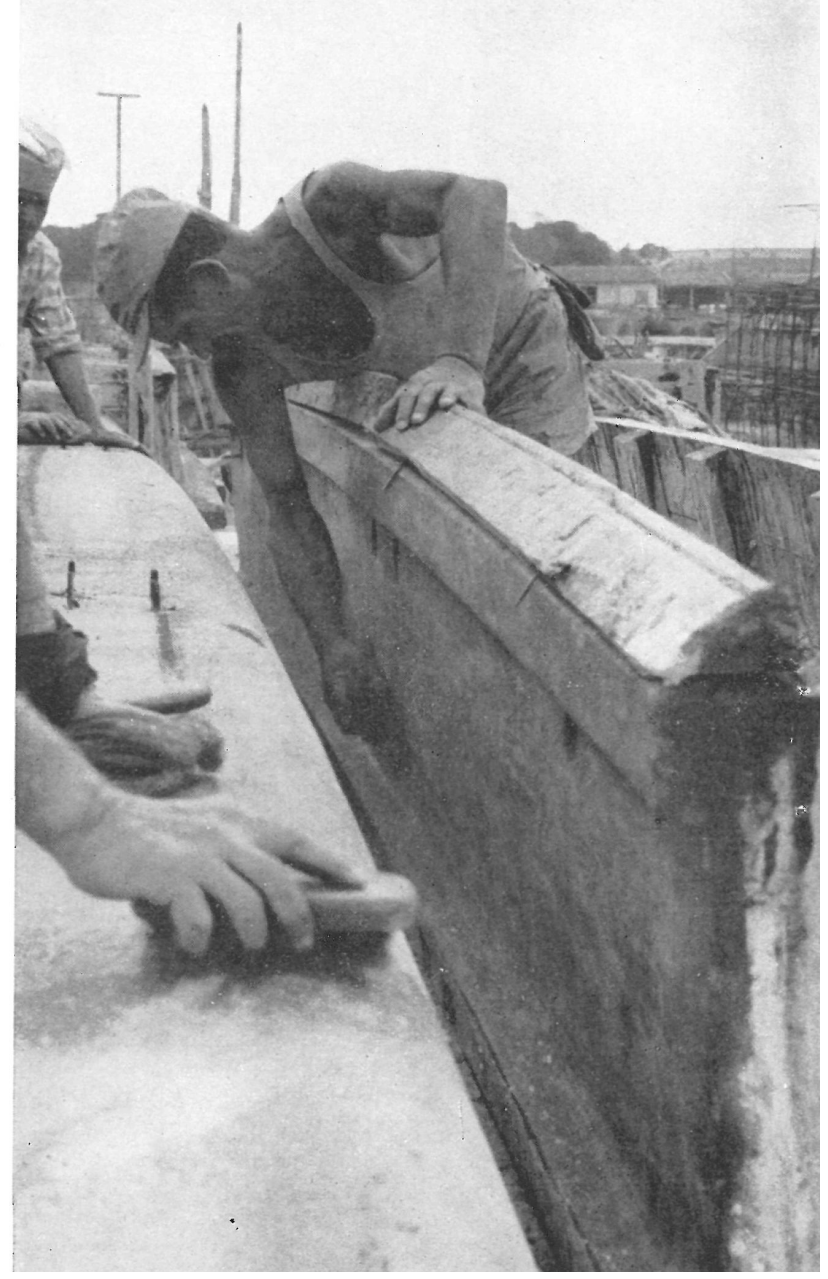




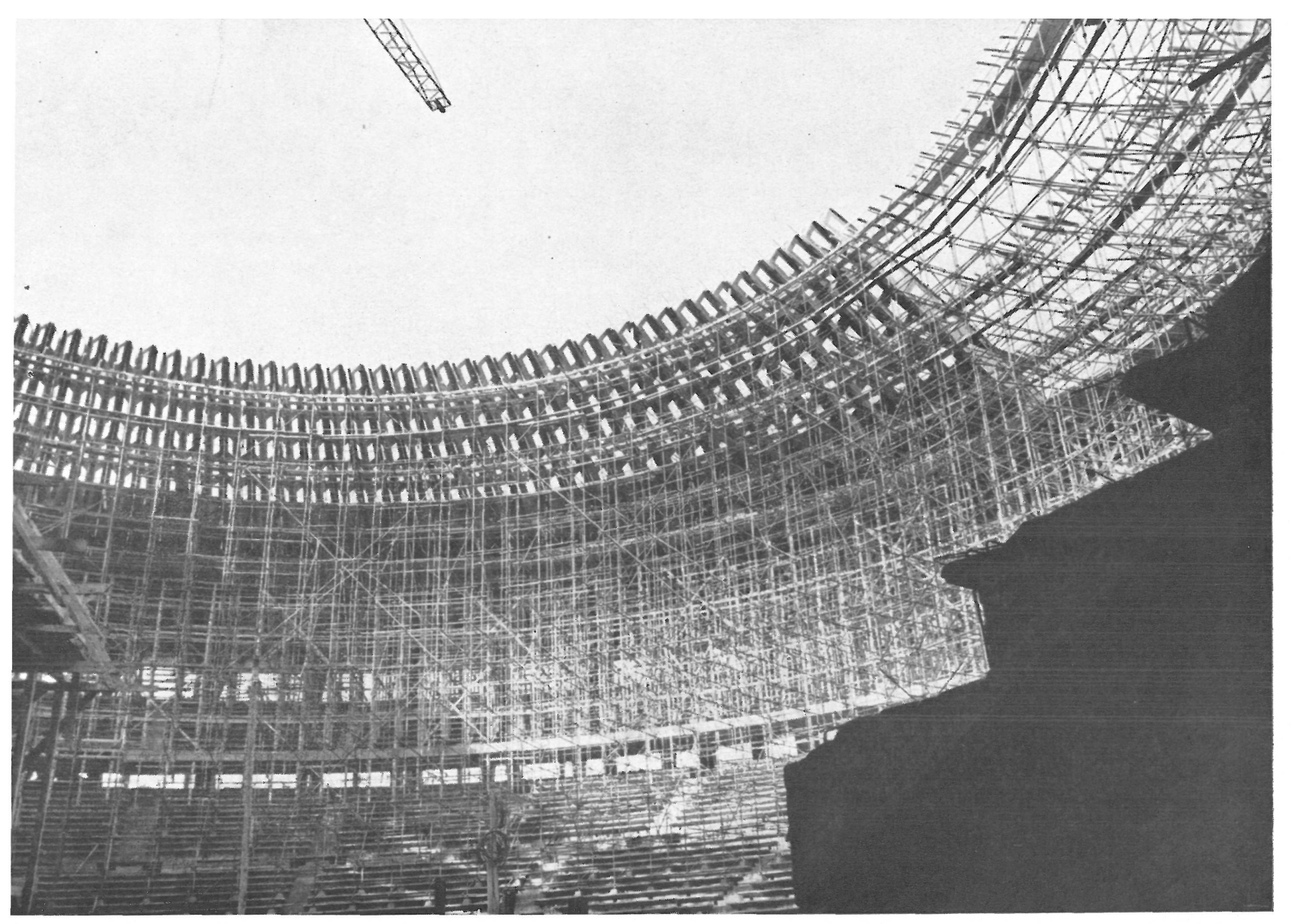

Con demasiada frecuencia este papel del ingeniero, de desarrollar una construcción honesta, formalmente limpia y audaz, no está bastante estimado por parte de los arquitectos, como lo demuestra la impresión de muchos ejemplos realizados y que siguen realizándose.

Generalmente, no se da bastante importancia al hecho de que la realización de formas libremente concebidas oponen al ingeniero problemas que exigen conocimientos teóricos de invención e intuición, que hacen de él un compañero de gran valor en la realización de un problema común. Pero el ingeniero tiene mucho que aprender y estar preparado para los grandes problemas que le plantean los arquitectos. Es totalmente falso el creer que el ingeniero sale de la escuela con los conocimientos suficientes para solucionar cualquier problema estático. Al contrario, la estática escolar abarca sólo un campo muy reducido, casi capítulos escogidos de un problema, que admite múltiples facetas dada la diversidad de formas posibles desde el punto de vista constructivo. Los deseos van siempre delante de la teoría, con pasos de gigante, como ocurre también en otros campos de la técnica.

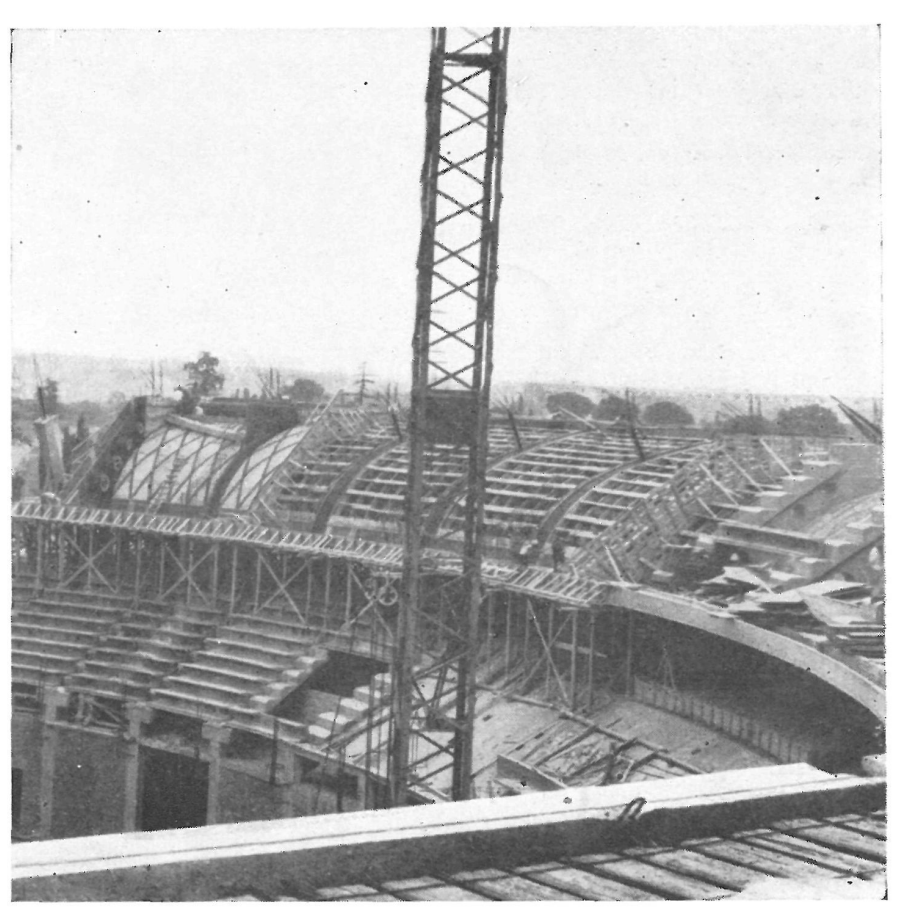




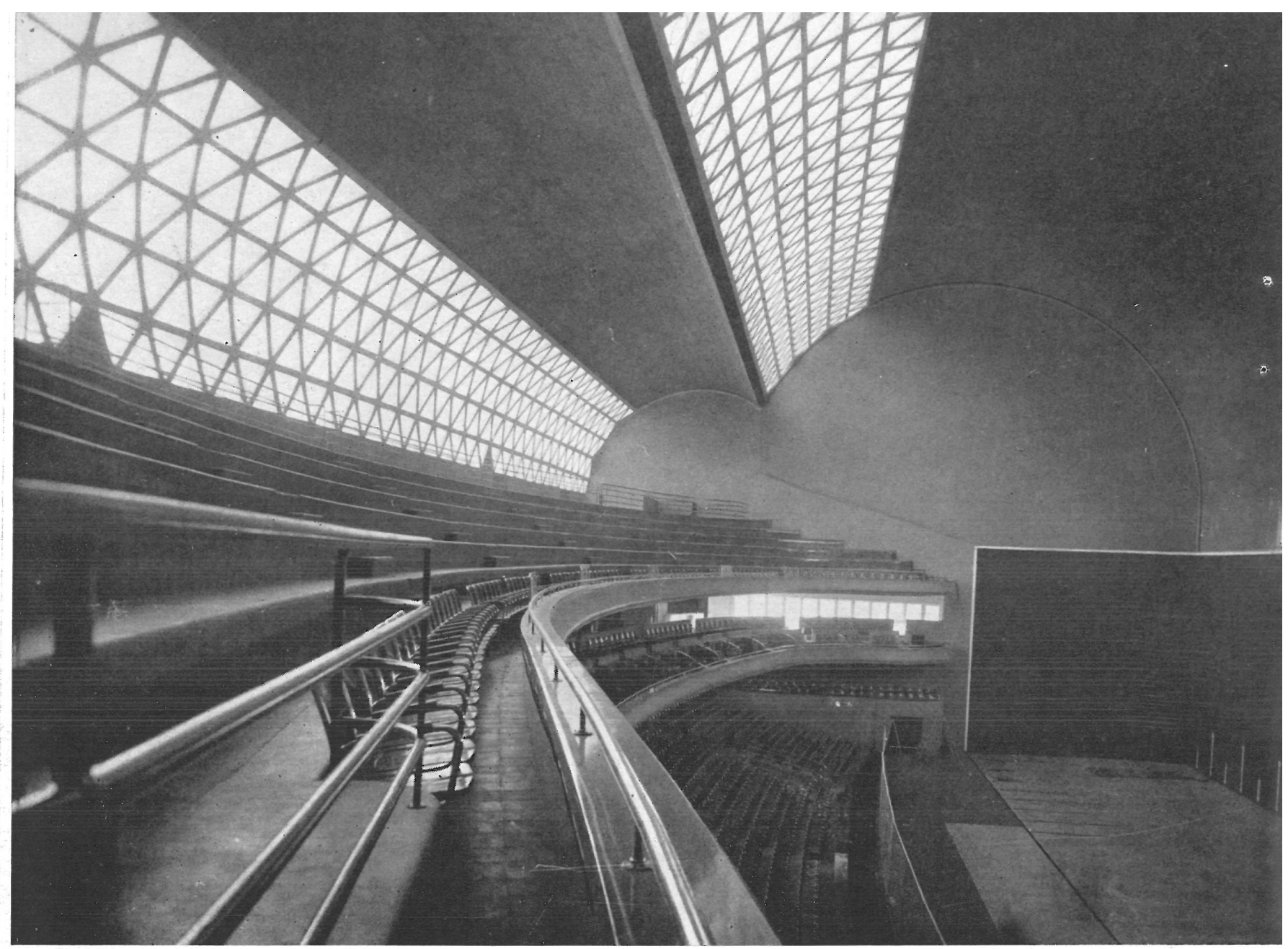

Eduardo Torroja:

Frontón Recoletos, Madrid.

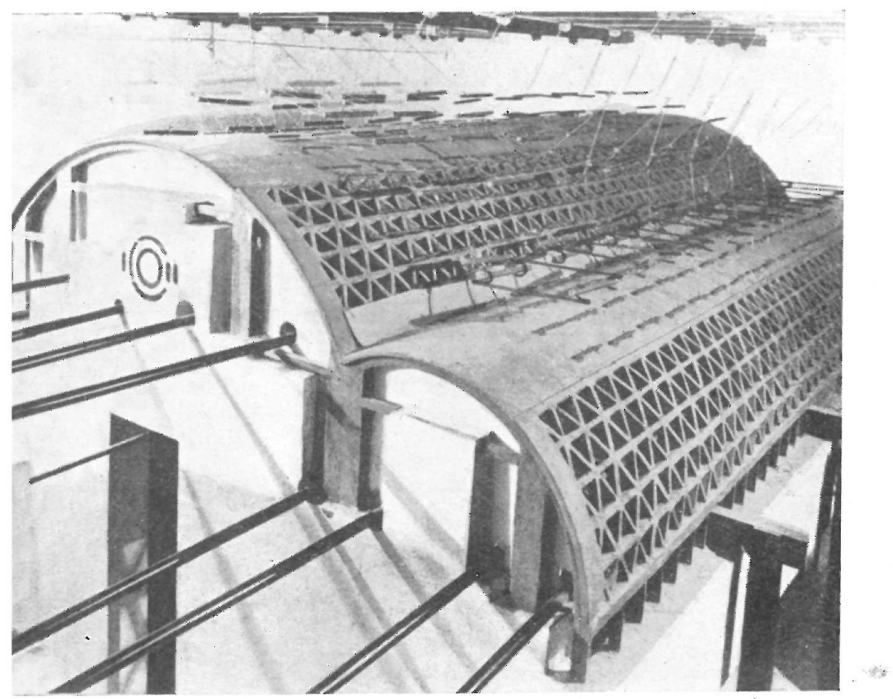

En este hecho, tanto el pensamiento del arquitecto como del ingeniero tienen que acostumbrarse. El ingeniero tiene que buscar personalmente caminos nuevos para realizar con la debida seguridad las nuevas formas de construcción que continuamente nacen, pues no queremos solamente construir lo que somos capaces de calcular, sino que tenemos que llegar a poder realizar todo aquello que deseamos construir.

A este respecto parece aleccionador el mostrar la forma de crear de tres ingenieros, cuyas obras están mundialmente reconocidas como formalmente ejemplares. Las obras de: Pier Luigi Nervi, Eduardo Torroja y Robert Maillart.

Ambos buscan la sencillez para la forma estructural y es un hecho evidente que ambos crean sus formas como consecuencia del profundo conocimiento intuitivo de las propiedades de los materiales, y nunca como consecuencia de un primer prejuicio estético. La arquitectura de estos ingenieros no es el resultado de ninguna "imaginación moderna". Nervi piensa, en primer lugar, como artesano, como constructor que ha creado durante décadas de años de trabajo agotador los métodos constructivos con cuya ayuda ahora estamos capacitados para poder realizar de forma correcta las construcciones de hormigón armado. 

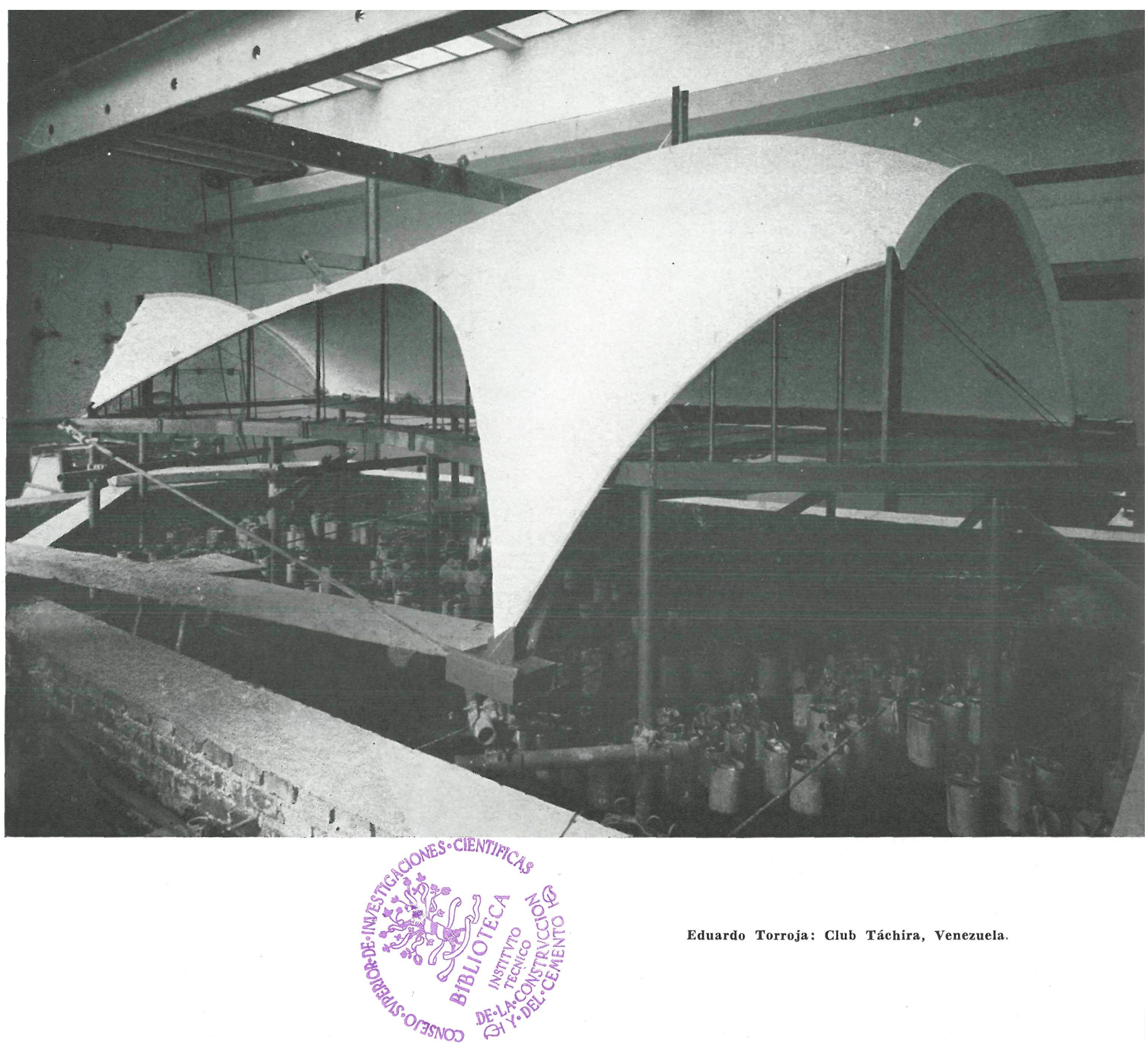

Eduardo Torroja: Club Táchira, Venezuela

Mediante la fina estructuración de su ferro-cemento, ha logrado liberar al hormigón de su elevado peso propio. En el camino de la realización de esta idea suya fundamental, ha sobrepasado innumerables dificultades técnicas y nos ha enseñado un camino enteramente nuevo en la forma de construir. No hace falta por ello enseñar aquí sus obras acabadas: son bastante conocidas a cada arquitecto. Pero algunas fotos de la obra, en construcción, de una de las nuevas instalaciones deportivas olímpicas de Roma-el Palacio de los Deportes-, darán una breve impresión de la variedad del minucioso trabajo técnico, cuya realización es decisiva para llegar a alcanzar la expresión grandiosa de los edificios de Nervi.

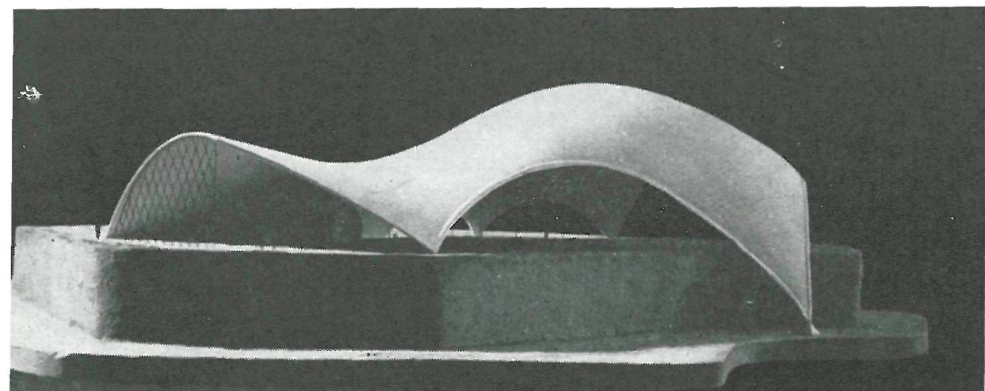

Eduardo Torroja toca el problema de la creación formal del material de otro lado. Es científico, inventor y, por eso, más libre de los métodos de ejecución. Pero cuanto más intensa ha sido su preocupación estática, mayores posibilidades ha descubierto en el campo de la concepción de nuevas formas constructivas. E ejemplo de un proyecto de bóveda laminar para un club en Venezuela, muestra la perfecta elegancia a la cual puede llegarse mediante una concepción libre, pero sujeta estática e intuitivamente. 